\title{
DC feedback for wide band frequency fixed current source
}

\author{
Aoday H. Al-Rawi ${ }^{1}$, W. M. A. Ibrahim ${ }^{1,2}$ and Eraj Humayun Mirza ${ }^{1}$ \\ 1. Department of Biomedical Engineering, University of Malaya, Kuala Lumpur, Malaysia \\ 2. E-mail any correspondence towmohdazhar@yahoo.com
}

\begin{abstract}
Alternating current sources are mainly used in bioelectrical impedance devices. Nowadays $50-100 \mathrm{kHz}$ bioelectrical impedance devices are commonly used for body composition analysis. High frequency bioelectrical impedance analysis devices are mostly used in bioimpedance tomography and blood analysis. High speed op-amps and voltage comparators are used in this circuit. Direct current feedback is used to prevent delay. An NChannel J-FET transistor was used to establish the voltage controlled gain amplifier (VCG). A sine wave signal has been applied as input voltage. The value of this signal should be constant in $170 \mathrm{mV} \mathrm{rms}$ to keep the output current in about $1 \mathrm{~mA}$ rms. Four frequencies; $100 \mathrm{kHz}, 1 \mathrm{MHz}, 2 \mathrm{MHz}$ and $3.2 \mathrm{MHz}$ were applied to the circuit and the current was measured for different load resistances. The results showed that the current was stable for changes in the resistor load, bouncing around an average point as a result of bouncing DC feedback.
\end{abstract}

Keywords: Bioimpedance analysis, wide band frequency, body composition, blood analysis, medical devices.

\section{Introduction}

Today bioelectrical impedance methods are widely used in many medical procedures such as bioelectrical impedance analysis (BIA) [1] and bioelectrical impedance tomography (EIT) [2-4]. BIA became very popular a few years ago for its ability to estimate the body composition [5]. EIT and BIA have since been expanded in scope to include measuring the respiratory rate and tissue state [6], the hematocrit $[7,8]$, and even the extent of dengue hemorrhagic fever in patients.

In the healthy living body, cell membranes consist of a layer of non-conductive lipid material sandwiched between two layers of conductive protein molecules. Biologically, the cell membrane functions as a permeable barrier separating the intracellular (cytoplasm) and extracellular components. The lipid membrane is traversed by proteins, which are soluble in water thus making pores through which water, ions, and other chemicals can enter and exit the cell. Controlling the flow of these materials is essential to life. The cell membrane protects the interior of the cell while allowing passage of some materials to which it is permeable. The cell membrane is composed mostly of a double layer of phospholipids, arranged tail to tail along the width of the cell membrane. This structure is called the lipid bilayer and is an electrical insulator (dielectric)

The impedance in human body cells varies from type to type and also with respect to input signal frequency. From the body equivalent circuit shown in Figure 1 [4] the total impedance of human tissue can be derived for both real (R) and imaginary $(\mathrm{X})$ parts as in equations 1 and 2:

$R=R_{o} \sqrt{\frac{R_{i}^{2}+X_{c}^{2}}{R_{i}^{2}+2 R_{i} R_{o}+R_{o}^{2}+X_{c}^{2}}} \cos \left(\tan ^{-1} \frac{X_{c}}{R_{i}}-\tan ^{-1} \frac{X_{c}}{R_{i}+R_{o}}\right)$

$X=R_{o} \sqrt{\frac{R_{i}^{2}+X_{c}^{2}}{R_{i}^{2}+2 R_{i} R_{o}+R_{o}^{2}+X_{c}^{2}}} \sin \left(\tan ^{-1} \frac{X_{c}}{R_{i}}-\tan ^{-1} \frac{X_{c}}{R_{i}+R_{o}}\right)$

Here $\mathrm{X}_{\mathrm{c}}=\frac{1}{2 \pi \mathrm{FC}}$ is the reactance which is frequency $(f)$ and capacitor $(C)$ dependent. For Figure $1, R_{\mathrm{o}}$ represents the extracellular resistance, $R_{\mathrm{i}}$ represents intracellular resistance, and $C_{\text {in }}$ is the cell membrane capacitance.

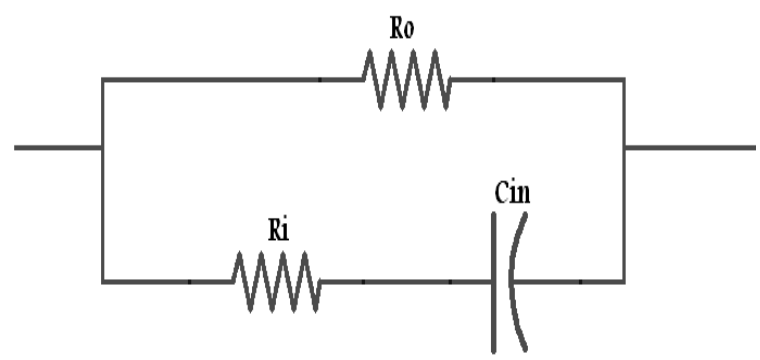

Fig. 1: Equivalent Electrical circuit model of the human body [5].

Equations 1 and 2 indicate that the reactance is inversely related to the frequency. The equivalent electrical circuit model of the human body as shown in Figure 1 reduces to a single resistor $R_{\mathrm{o}}$ for frequencies below $50 \mathrm{kHz}$. At higher frequencies the capacitor acts as short circuit so the equivalent circuit of the cell will be the two resistors $R_{\mathrm{i}}$ and $R_{\mathrm{o}}$ in parallel. Since alternating current (AC) is used, many different techniques are used in BIA depending on the frequency. Because of the varying in tissue membrane properties this make the membrane's capacitance differ for each tissue type [9].

The circuit of Figure 1 yields both real and imaginary components (equations 1 and 2), with both components experiencing a frequency dependence shown in Figure 2. Wide band frequency fixed current is required to obtain impedance information from the human tissue.

There are many current circuits that can be used. Amongst them are the well-known current pump, voltageto-current, and fixed current circuits. All these circuits serve the same function to feed fixed current to a load. Typically AC fixed current injecting circuits with current between $500 \mu \mathrm{A}$ and $1 \mathrm{~mA}$ are normally used in BIA and BIT applications. 


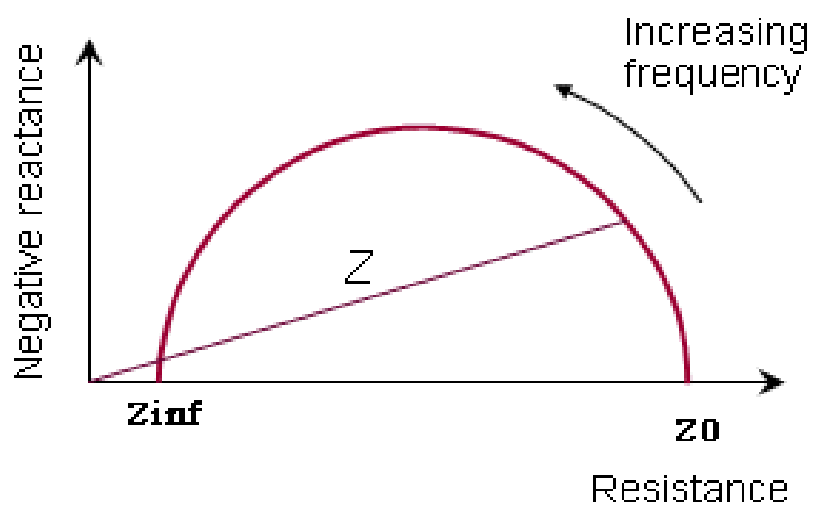

Fig. 2: Cole Plot [9]

Bragos et al.[3] has built a current source by using three operational amplifiers for current up to $1 \mathrm{MHz}$. Shuai et al. [11] and Halter et al. [4] have built complete bioimpedance systems for EIT using enhanced Howland current sources. Cheng et al. [12] and Ross et al. [13] built BIA systems using enhanced Howland current sources. Terzopoulos et $a l$. [14] used a current mirror circuit to achieve the target. Seoane et. al [15] used only one amplifier with a floating, rather than grounded, load. Bertemes-Filho et al. [16] has made a comparison between the Howland circuits and current mirror type and concluded that both circuits failed to achieve 100 kilohm output impedance at $1 \mathrm{MHz}$ frequency. The Howland current pump is a stable circuit for current up to $1 \mathrm{MHz}$ but needs very accurately balanced bridge resistors [17].

\section{Materials and methods}



Fig. 3: Block Diagram for the complete system

Figure 3 illustrates the block diagram of the complete system used for the current source. The voltage controlled gain amplifier is designed using a high-speed amplifier with an N-Channel JFET transistor shown in Figure. 4. A THS 4011 high-speed amplifier with bandwidth from DC up to $350 \mathrm{MHz}$ is used to control the signal amplitude depending on the load current. Different gain can be obtained by controlling the DC voltage on the gate of the JFET transistor. The relation between the gate voltage and the amplifier gain is shown in Figure 5.
The final relation between the feedback voltage and the operational amplifier is linear because the gain in the JFET is exponential and for the operational amplifier is $f(1 / x)$. After multiplying the two gains the final model will be linear within a certain range (the relation between the Vgs and Id).

A shunt resistor $\left(R_{S}\right)$ in series with the load acts as a current sensor. An AD8130 differential amplifier was used to amplify the voltage across the $\mathrm{R}_{\mathrm{S}}$. A basic envelope demodulator was used to convert the $\mathrm{AC}$ output voltage to DC voltage. Figure 4 shows the schematic diagram for both the differential amplifier and envelope circuit.

An LM393 voltage comparator was used to compare the output DC voltage from the AD8130 differential opamp (I to $\mathrm{V}$ circuit) with the reference voltage. This reference voltage was used to limit the load current. The basic envelope demodulator was used with a reversed connection to generate negative voltage as shown in Figure 4.

\section{The circuit's function}

The proposed circuit has been built on basic VCG. The load variation will control the amplifier gain to generate a fixed current. When the load impedance is decreased the load current will increase. The increase in load current leads the voltage across the $\mathrm{R}_{\mathrm{S}}\left(\mathrm{R}_{4}\right.$ in Figure 4$)$ in the current sensor to increase. This voltage is multiplied by the gain of the amplifier and is rectified in the envelope demodulator (diode, capacitor, and resistor). Finally this signal is compared with a certain level of DC voltage. The value of this voltage will limit the load current to the required value. The output of the comparator circuit is a square pulse width modulated waveform. This waveform is applied to a half bridge rectifier to provide negative voltage. Finally this voltage is directed to the FET transistor gate and vice versa.

\section{Results and discussion}

The final result for this circuit shows that we can achieve a fixed current output (figure 6). The comparator waveforms output signal and the current output signal is shown in Figure 7. As we mentioned in the methodology there is only one major drawback to this circuit and it is that the output current signal is not 100 percent fixed. There are a few ripples in the amplitude. Theses ripples are due to the comparator output which affects the gate of the JFET transistor and the amplifier gate. Table 1 is an example of the result of the load resistance against the load current at 2 MHz. The results showed that the load current was stable against the changing load resistance. It is also found that the load current was stable against the load impedance until about $5 \mathrm{MHz}$ except the upper limit for the load resistor went down to $1900 \mathrm{ohm}$. 


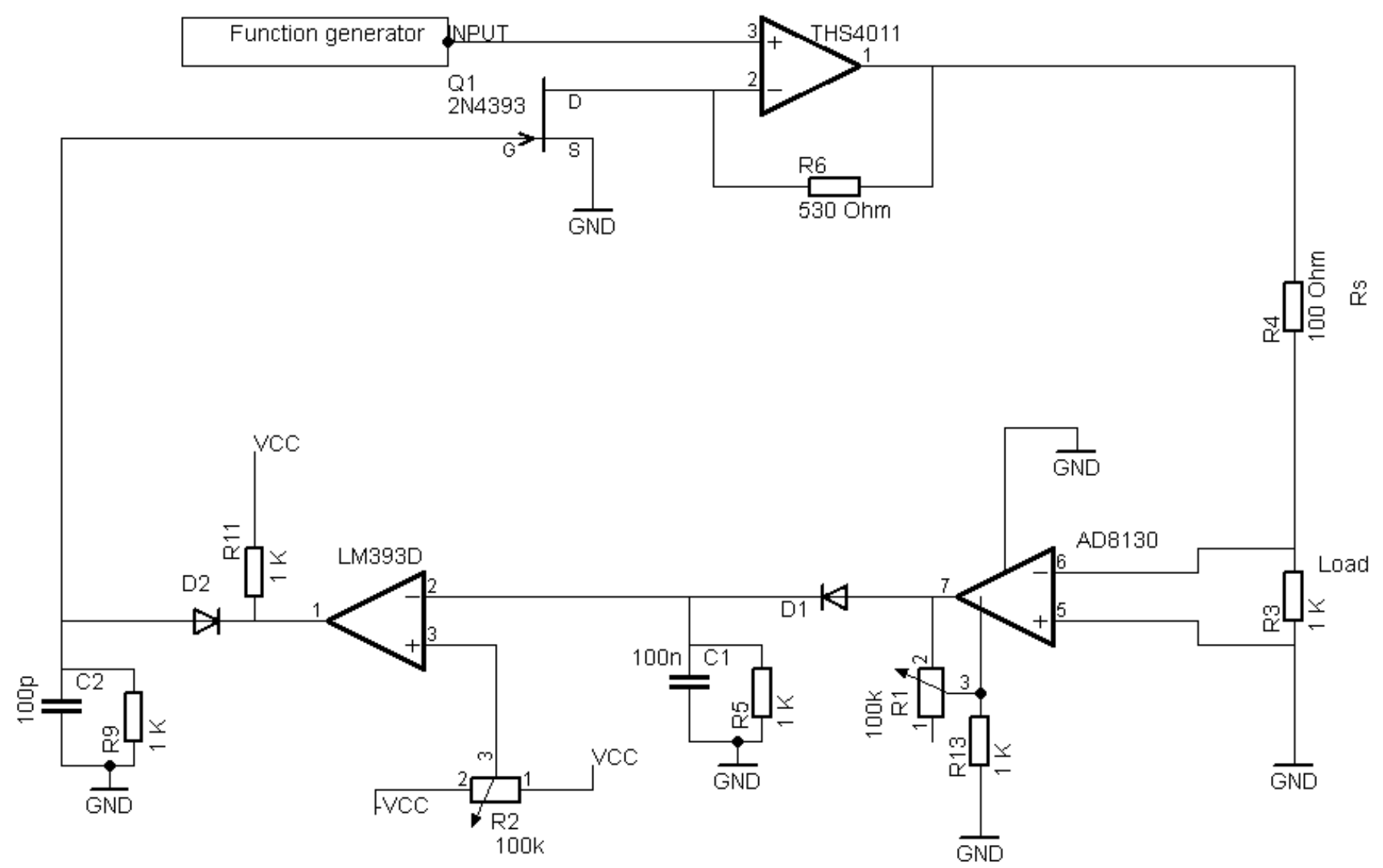

Fig. 4: The voltage gained controlled

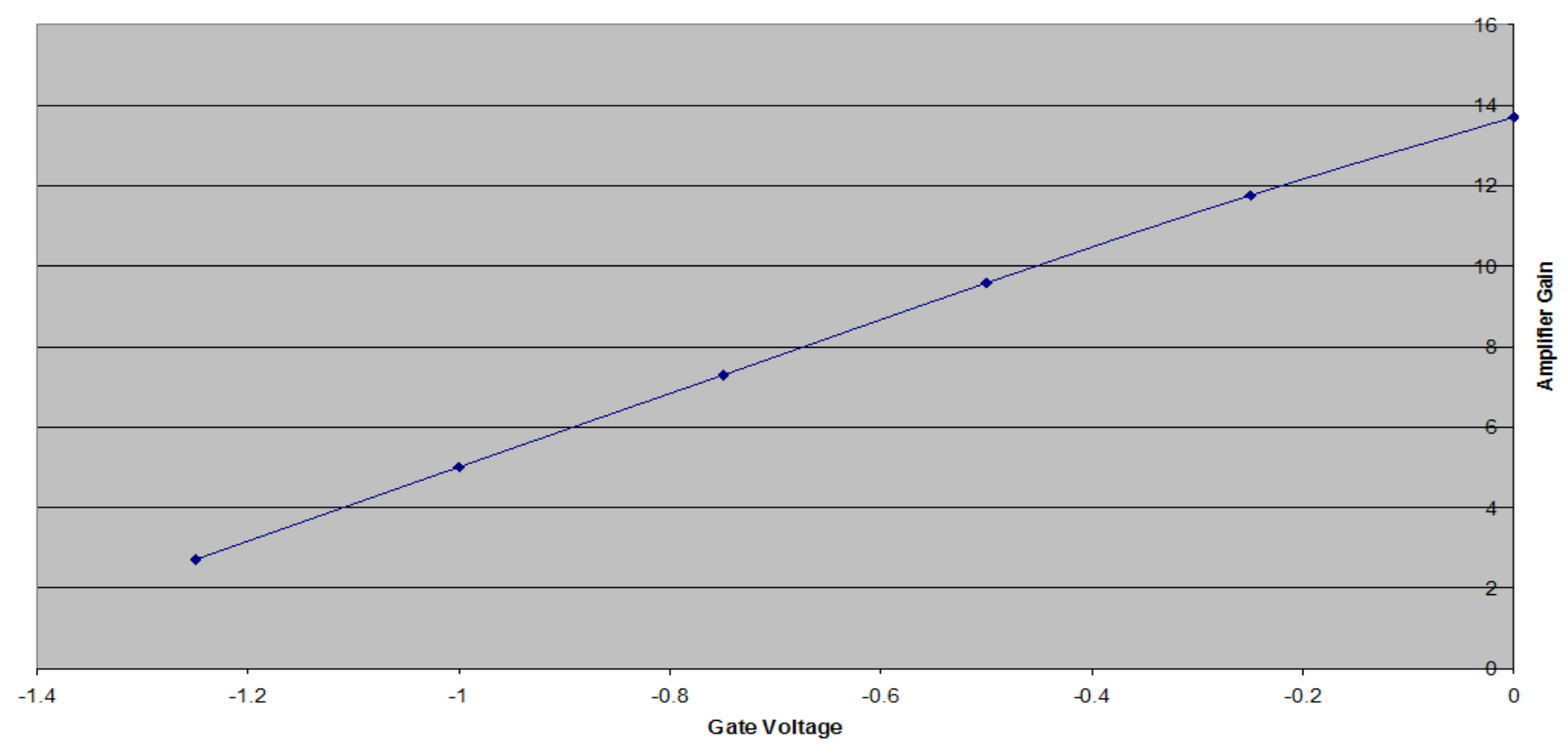

Fig. 5: The effect of gate voltage on amplifier gain. 
A high frequency current source is required in many bioimpedance applications such as (BIA and EIT). Many researchers have designed high frequency current sources with different methods like the Howland and the floating load circuits. In this study a DC feedback method was used to achieve higher frequencies for BIA measurements. This is applied to overcome the delay effect in the signal at high frequencies. Whereas a VCG amplifier is used to control the gain depending on the gain of the amplifier circuit, here the response for this circuit depended on the response of the J-FET transistor, with Rf/Rjfet being the amplifier gain where the Rjfet is exponentially changed against the Vgs.
The overall equation controls the gain that gave linear DC input voltage. There are two important parts in the design. The first is shown in Figure 6, where the current through the load is sensed and converted into an amplified voltage, and then apply the envelope circuit to change it to DC. The second is that the DC voltage is compared to a reference voltage to generate the pulse width modulated signal to apply to the J-FET transistor. The comparator speed is a very significant factor. All the diodes here are Schottky diodes that give very low voltage drop. The main shortage for this circuit is that the output current and voltage has a ripple.

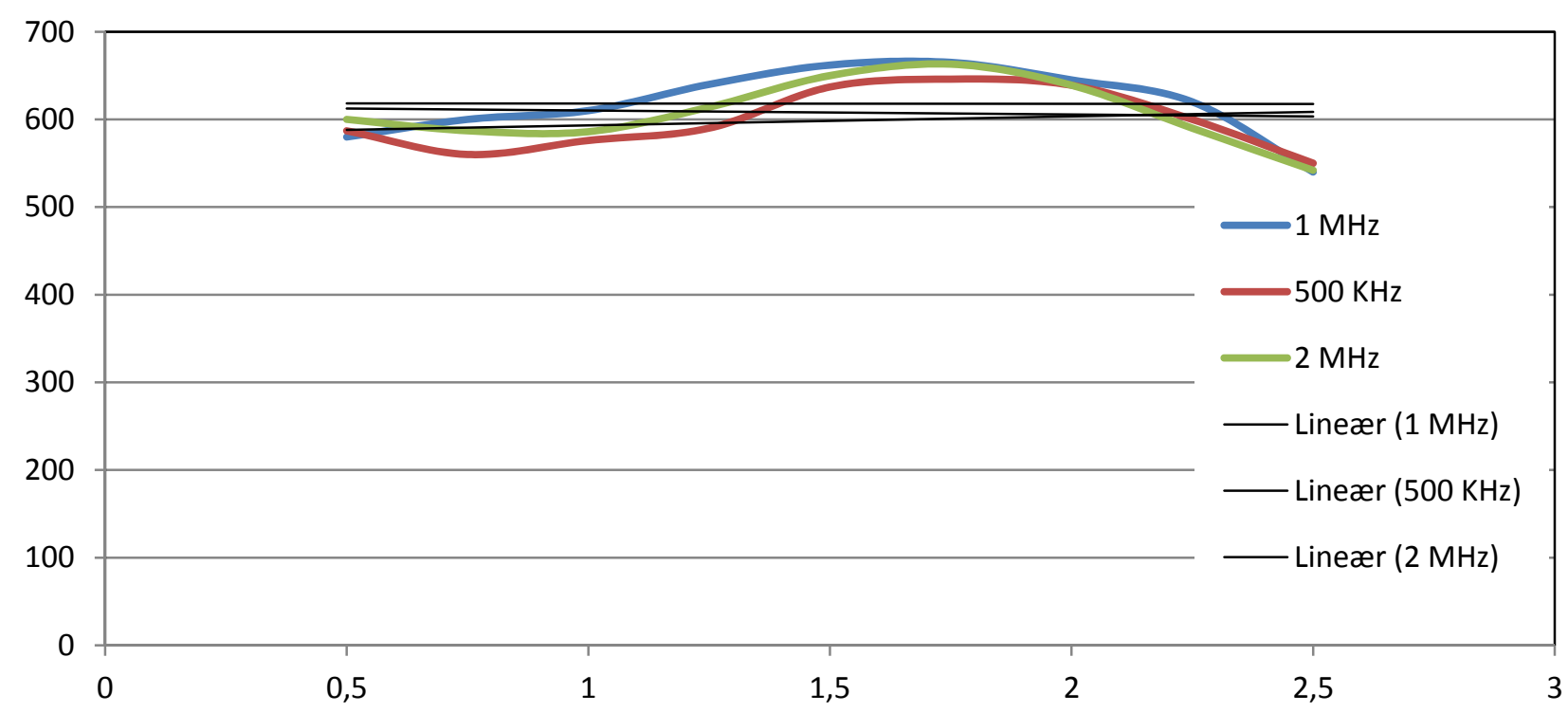

Fig. 6: The current in $\mathrm{mA}$ against the load resistance in $\mathrm{k} \Omega$

\begin{tabular}{cc}
\hline $\begin{array}{c}\text { load } \\
\mathrm{k} \Omega\end{array}$ & $\begin{array}{c}\text { current } \\
\mathrm{mA}\end{array}$ \\
\hline 0.5 & 600 \\
0.75 & 587 \\
1 & 586 \\
1.25 & 614 \\
1.5 & 650 \\
1.75 & 663 \\
2 & 639 \\
2.25 & 590 \\
2.5 & 542 \\
\hline
\end{tabular}

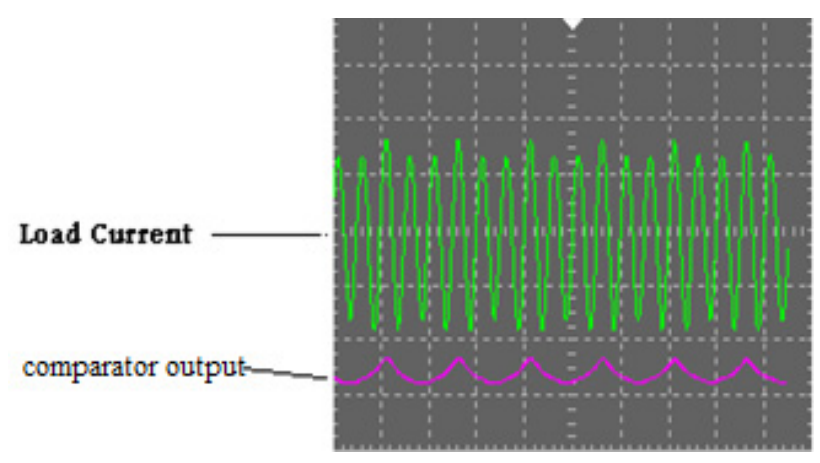

Table 1: The load against the current with $2 \mathrm{MHz}$

Fig. 7: Waveforms of comparator and Load current 


\section{References}

1. R. J. Liedtke, "The Fundamentals of Bioelectrical Impedance Analysis", http://rjlsystems.com, February 1, 1998.

2. D. S. Holder, "Electrical Impedance Tomography, Methods, History and Applications", Institute of Physics Publishing, 2005.

3. R. Bragos, J. Rosell, and P. Riu, "A wide-band AC-coupled current source for electrical impedance tomography", Physiol. Meas. 15, A91-A99, 1994. http://dx.doi.org/10.1088/0967-3334/15/2A/013

4. R. Halter, A. Hartov, and Keith D Paulsen, "Design and implementation of a high frequency electrical impedance tomography system" Physiol. Meas. 25, 379, 2004. http://dx.doi.org/10.1088/0967-3334/25/1/041

5. F. Ibrahim, M. N. Taib, W. A. B. Wan Abas, C. C. Guan, and S. Sulaiman," A novel approach to classify risk in dengue hemorrhagic fever (DHF) using bioelectrical impedance analysis (BIA)", IEEE Trans. Instrument. Meas., 54(1), 237244, 2005. http://dx.doi.org/10.1109/TIM.2004.840237

6. R.F. Kushner, "Bioelectrical impedance analysis: A review of principles and applications," J. Am. Col. Nutr., 2(11), 199209, 1992.

7. F. Jaspard, M. Nadi, and A. Rouane, "Dielectric properties of Blood: An investigation of haematocrit dependence".

Physiol. Meas. 24, 137-147, 2003. http://dx.doi.org/10.1088/0967-3334/24/1/310

8. F. Ibrahim, N. A. Ismail, M. N. Taib, W. A. B. Wan Abas, S. Sulaiman, and C. C. Guan, "Assessment of haematocrit status using bioelectrical impedance analysis in dengue patients", IFAC Modeling and Control in Biomedical Systems, Melbourne, Australia, 2003.
9. A. H. Ar-Rawi, M. Moghavvimi, and W. Ibrahim, "Novel idea to monitor and measure blood hemoglobin noninvasively", African J. Biotech., 9(54), 9295-9306, 2010.

10. K. S. Cole and R. H. Cole. "Dispersion and absorption in dielectrics - I. alternating current characteristics". Journal of Chemical Physics, 9, 341-251, 1941. http://dx.doi.org/10.1063/1.1750906

11. Z. Shuai, X. Guizhi, W. Huanli, G. Duyan, and Y. Weili, "Multi-frequency EIT Hardware System Based on DSP", IEEE International Conference of the EMBS, 2006.

12. K. S. Cheng, C. Y. Chen, M. W. Huang, and C. H. Chen, " A Multi-Frequency Current Source For Bioimpedance Application", 5th International IEEE EMBS Special Topic Conference on Information Technology in Biomedicine, 2006

13. A. S. Ross, G. J. Saulnier, J. C. Newell, and D. Isaacson, "Current source design for electrical impedance tomography", Physiol. Meas., 24, 509-516, 2003.

14. N. Terzopoulos, K. Hayatleh, B. Hart, F. J. Lidgey, and C. McLeod, "A novel bipolar-drive circuit for medical applications". Physiol. Meas., 26, N21-N27, 2005.

15. F. Seoane, R. Bragos, and K. Lindecrantz, "Current source for multi frequency broadband electrical Bioimpedance spectroscopy systems. A Novel Approach", IEEE International Conference of the EMBS, 2006.

16. P. Bertemes - Filho, B. H. Brown, and A. J. Wilson, "A comparison of modified Howland circuits as current generator with current mirror type circuits", Physiol. Meas., 21, 1-6, 2000. http://dx.doi.org/10.1088/0967-3334/21/1/301

17. A. Robert, "A Comprehensive Study of the Howland Current Pump", National Semiconductor Application Note 1515. January 29, 2008. 